\title{
Calculation of the magnetic hyperfine structure constant of alkali metals and alkaline earth metal ions using the relativistic coupled-cluster method
}

\author{
Sudip Sasmal* \\ Electronic Structure Theory Group, Physical Chemistry Division, \\ CSIR-National Chemical Laboratory, Pune, 411008, India
}

\begin{abstract}
The Z-vector method in the relativistic coupled-cluster framework is used to calculate magnetic hyperfine structure constant $\left(A_{J}\right)$ of alkali metals and singly charged alkaline earth metals in their ground state electronic configuration. The Z-vector results are in very good agreement with the experiment. The $A_{J}$ values of $\mathrm{Li}, \mathrm{Na}, \mathrm{K}, \mathrm{Rb}, \mathrm{Cs}, \mathrm{Be}^{+}, \mathrm{Mg}^{+}, \mathrm{Ca}^{+}$, and $\mathrm{Sr}^{+}$obtained in the Z-vector method are compared with the extended coupled-cluster results taken from Phys. Rev. A 91, 022512 (2015). The same basis and cutoff are used for the comparison purpose. The comparison shows that Z-vector method with the singles and double approximation can produce more precise wavefunction in the nuclear region than the ECC method.

PACS numbers: 31.15.aj, 31.15.am, 31.15.bw
\end{abstract}

\section{INTRODUCTION}

The interaction of electromagnetic field of electrons with the nuclear moments of nucleus, known as hyperfine structure interaction, causes small shift and splitting of energy levels [1]. Therefore, it is very important for the accurate description of energy levels of atom, molecule and ion. The precise measurements of the energy levels of alkali metals play important role in various areas of atomic and nuclear physics as they are extensively used in high precession spectroscopy, laser cooling and trapping of atom, ultracold collision studies, photo-association spectroscopy, Bose-Einstein condensation and more recently, test for parity and time reversal violation. Currently, the hyperfine transition of Cs atom $\left[[\mathrm{Xe}] 6 \mathrm{~S}\left({ }^{2} S_{1 / 2}, F=3, m_{F}=0\right) \leftrightarrow[\mathrm{Xe}] 6 \mathrm{~S}\left({ }^{2} S_{1 / 2}, F=\right.\right.$ $\left.\left.4, m_{F}=0\right)\right]$ is used as frequency standard which is accurate up to 1 per $10^{15}$ [2]. Singly ionized alkaline earth metal ions are insensitive to the perturbation of the environment arising form collisions and Doppler shift and thus, have been considered for the potential candidates for optical frequency standard [3-6]. The ${ }^{2} S_{1 / 2}$ ground state of these ions are regarded for quantum information processing studies to encode quantum-bit into hyperfine levels because of their long phase coherence due to their small energy gap and relatively large spontaneous decay lifetime $[7,8]$.

Recently, experiments for parity non-conservation (PNC) become a cutting-edge topic as it can test the accuracy of fundamental physics and explore "new physics" beyond the standard model. However, the PNC amplitudes, which are very essential to determine the value of PNC constants cannot be measured experimentally and thus, has to be obtained theoretically. Therefore, it is extremely important to have a reliable way of determining the accuracy of such theoretical calculations. The PNC

*sudipsasmal.chem@gmail.com amplitudes are very sensitive to the accuracy of the wavefunction in the near nuclear region $[9,10]$. The same is true for HFS constants [11]. Therefore, one can assess the accuracy of PNC amplitudes by comparing theoretically obtained HFS constants with corresponding experimental values $[12-14]$.

Relativistic effects are very important for the precise calculation of the wavefunction in the near nuclear region. For a single determinant theory, the best way to include relativistic effect is to solve the four-component Dirac-Hartree-Fock (DHF) equation. However, the DHF method misses the instantaneous interaction of opposite spin electrons. Coupled-cluster (CC) [15-17] is the most elegant method to include this dynamic electron correlation.

The coupled-cluster equation can be solved either by variationally or by non-variationally. Although, the nonvariational coupled-cluster, also known as normal coupled cluster (NCC) is the most familiar, the variational coupled-cluster (VCC) has several advantages over the NCC. The VCC, being variational has upper boundedness in energy and satisfies the generalized HellmannFeynman (GHF) theorem which simplifies the calculations for higher order properties. Unitary coupled-cluster (UCC) [18-23], expectation value coupled-cluster (XCC) [24-27] and extended coupled-cluster (ECC) $[28,29]$ are the most familiar VCC [30] in literature. Recently, ECC has been extended to the relativistic regime to calculate magnetic HFS constants of atoms and molecules [31]. ECC uses dual space of right and left vectors in a double linked form where the left vector is not complex conjugate of the right vector. Although ECC functional is a terminating series, the natural termination leads to very expensive terms. Thus, for practical purpose, one needs to use some truncation scheme to avoid computationally expensive terms.

On the other hand, the NCC is nonvariational and thus, does not satisfy the GHF theorem. Therefore, the expectation value and first order energy derivative yield different results $[32,33]$. However, the energy derivative 
method is superior than the expectation value method as the property value obtained in energy derivative method can be expressed as the corresponding expectation value plus some additional terms which make it closer to the full configuration interaction property value. The NCC energy is not optimized with respect to determinantal coefficients $\left(C_{d}\right)$ in the expansion of many electron wavefunction [32]. Thus, the derivative of energy with respect to external perturbation requires the derivative of energy with respect to $C_{d}$ times the derivative of $C_{d}$ with respect to external perturbation. The derivative terms involving $C_{d}$ can be included in Z-vector $[34,35]$ method by introducing a perturbation independent de-excitation operator where the equation for this operator is linear. Thus, for any number of property calculation, one needs to calculate only one set of coupled-cluster amplitudes. The advantage of Z-vector method over ECC method is that unlike ECC, the equations for excitation operators are decoupled from the de-excitation operators. This saves enormous computational cost. Recently, Z-vector method is extended to the relativistic region for the calculation of ground state properties of atomic and molecular systems [36].

In this paper, we have calculated the magnetic HFS constant of alkali metal atoms and singly charged alkaline earth metal cations using Z-vector technique in the relativistic coupled-cluster framework. We have compared the Z-vector values with the ECC values calculated in Ref. [31] to show that the Z-vector method with single and double approximation can produce much better wavefunction in the nuclear region of atomic nucleus than the ECC method with the approximation stated in the paper and thus, is capable of providing the precise value of the types of property like PNC amplitudes, which are prominent in the nuclear region. The paper is organize as follows. A brief introduction and the workable equations for the Z-vector method are given in Sec. II followed by the matrix elements for the magnetic HFS constant of atomic system in the same section. The computational details are given in Sec. III. In Sec. IV, we present our results and discuss about those before making our final remarks in Sec. V.

\section{THEORY}

\section{A. Z-vector method}

The study of hyperfine interaction helps us to understand nuclear structure of an atom and its impact on the electronic wavefunction in the nuclear and near nuclear region. Therefore, for the accurate calculation of magnetic HFS constant, which demands very precise wavefunction in the short range of nucleus, we need to incorporate both relativistic and electron correlation effects. In this work, the four component Dirac-HartreeFock (DHF) method is used to include the effect of relativity where the electron-electron repulsion term is ap- proximated as Coulomb interaction. The Dirac-Coulomb Hamiltonian is given by

$$
\begin{aligned}
H_{D C}= & \sum_{i}\left[-c(\vec{\alpha} \cdot \vec{\nabla})_{i}+\left(\beta-\mathbb{1}_{4}\right) c^{2}+V^{n u c}\left(r_{i}\right)+\right. \\
& \left.\sum_{j>i} \frac{1}{r_{i j}} \mathbb{1}_{4}\right]
\end{aligned}
$$

where, $\alpha$ and $\beta$ are the usual Dirac matrices, $c$ is the speed of light, $\mathbb{1}_{4}$ is the $4 \times 4$ identity matrix and $V^{n u c}\left(r_{i}\right)$ is the nuclear potential function and the Gaussian charge distribution is used in this work. The DHF method misses the instantaneous dynamic correlation of opposite spin electrons. Among various many-body theory, the single reference coupled-cluster (SRCC) is the most elegant technique to incorporate dynamic correlation. The SRCC wavefunction is given as

$$
\left|\Psi_{c c}\right\rangle=e^{T}\left|\Phi_{0}\right\rangle,
$$

where, $\Phi_{0}$ is the DHF wavefunction and $T$ is the coupledcluster excitation operator which is given by

$$
T=T_{1}+T_{2}+\cdots+T_{N}=\sum_{n}^{N} T_{n},
$$

with

$$
T_{m}=\frac{1}{(m !)^{2}} \sum_{i j \ldots a b \ldots} t_{i j \ldots}^{a b \ldots} a_{a}^{\dagger} a_{b}^{\dagger} \ldots a_{j} a_{i} .
$$

Here, i,j(a,b) are the hole(particle) indices and $t_{i j . .}^{a b .}$ are the cluster amplitudes corresponding to the cluster operator $T_{m}$. In the coupled-cluster single and double (CCSD) approximation, $T=T_{1}+T_{2}$. The equations for $T_{1}$ and $T_{2}$ are given as

$$
\begin{gathered}
\left\langle\Phi_{i}^{a}\left|\left(H_{N} e^{T}\right)_{c}\right| \Phi_{0}\right\rangle=0, \\
\left\langle\Phi_{i j}^{a b}\left|\left(H_{N} e^{T}\right)_{c}\right| \Phi_{0}\right\rangle=0,
\end{gathered}
$$

where, $\mathrm{H}_{N}$ is the normal ordered DC Hamiltonian and subscript $c$ means only the connected terms exist in the contraction between $\mathrm{H}_{N}$ and T. Size-extensivity is ensured by this connectedness. The coupled-cluster correlation energy can be obtained as

$$
E^{c o r r}=\left\langle\Phi_{0}\left|\left(H_{N} e^{T}\right)_{c}\right| \Phi_{0}\right\rangle .
$$

However, the SRCC energy is not optimized with respect to the determinantal coefficients and the molecular orbital coefficients in the expansion of the many electron correlated wavefunction [32]. Therefore, the calculation of SRCC energy derivative with respect to external perturbation requires to include these derivative terms. The equation for these terms are linear but in general, perturbation dependent. However, in Z-vector method, the derivative terms containing the determinantal coefficients can be incorporated by the introduction of a perturbation independent operator $\Lambda$ [35]. Thus, in the Z-vector 
method, any number of property calculations can be done by solving only one set of $T$ and $\Lambda$ amplitudes. $\Lambda$ is a deexcitation operator and the second quantized form is given by

$$
\Lambda=\Lambda_{1}+\Lambda_{2}+\ldots+\Lambda_{N}=\sum_{n}^{N} \Lambda_{n}
$$

where,

$$
\Lambda_{m}=\frac{1}{(m !)^{2}} \sum_{i j . . a b . .} \lambda_{a b . .}^{i j . .} a_{i}^{\dagger} a_{j}^{\dagger} \ldots a_{b} a_{a} .
$$

Here $\lambda_{a b . .}^{i j . .}$ are the cluster amplitudes corresponding to the cluster operator $\Lambda_{m}$. In CCSD approximation, $\Lambda=$ $\Lambda_{1}+\Lambda_{2}$. The explicit equations for the amplitudes of $\Lambda_{1}$ and $\Lambda_{2}$ operators are given by

$$
\begin{gathered}
\left\langle\Phi_{0}\left|\left[\Lambda\left(H_{N} e^{T}\right)_{c}\right]_{c}\right| \Phi_{i}^{a}\right\rangle+\left\langle\Phi_{0}\left|\left(H_{N} e^{T}\right)_{c}\right| \Phi_{i}^{a}\right\rangle=0 \\
\left\langle\Phi_{0}\left|\left[\Lambda\left(H_{N} e^{T}\right)_{c}\right]_{c}\right| \Phi_{i j}^{a b}\right\rangle+\left\langle\Phi_{0}\left|\left(H_{N} e^{T}\right)_{c}\right| \Phi_{i j}^{a b}\right\rangle \\
+\left\langle\Phi_{0}\left|\left(H_{N} e^{T}\right)_{c}\right| \Phi_{i}^{a}\right\rangle\left\langle\Phi_{i}^{a}|\Lambda| \Phi_{i j}^{a b}\right\rangle=0
\end{gathered}
$$

The energy derivative is given by

$$
\Delta E^{\prime}=\left\langle\Phi_{0}\left|\left(O_{N} e^{T}\right)_{c}\right| \Phi_{0}\right\rangle+\left\langle\Phi_{0}\left|\left[\Lambda\left(O_{N} e^{T}\right)_{c}\right]_{c}\right| \Phi_{0}\right\rangle .
$$

Here, $O_{N}$ is the derivative of normal ordered perturbed Hamiltonian with respect to external field of perturbation. It is clear from the above formulation that the derivative terms containing only the determinantal coefficients are included here, i.e., the orbital relaxation terms that are required to make energy functional stationary with respect to molecular orbital coefficients are not considered here. It is worth to mention that recently, Saue and coworkers [37] have implemented the orbitalunrelaxed analytical method in the four-component relativistic SRCC framework based on the Lagrangian multiplier method of Helgaker and coworkers [38] which is similar to the Z-vector method for the ground state first order properties.

\section{B. Magnetic hyperfine structure constant}

The magnetic HFS interaction arises due to the coupling of nuclear magnetic moment with the angular momentum of electrons and thus, can be treated as a onebody interaction from the electronic structure point of view. The magnetic vector potential due to a nucleus is given by

$$
\vec{A}=\frac{\vec{\mu}_{k} \times \vec{r}}{r^{3}}
$$

where, $\vec{\mu}_{k}$ is the magnetic moment of nucleus $K$. In Dirac theory, the HFS interaction Hamiltonian due to $\vec{A}$ can be given as

$$
H_{\mathrm{hfs}}=\sum_{i}^{n} \vec{\alpha}_{i} \cdot \vec{A}_{i}
$$

where, $\alpha_{i}$ denotes the Dirac $\alpha$ matrices for the $\mathrm{i}^{\text {th }}$ electron and $n$ is the total no of electrons. The magnetic hyperfine constant of the $J^{t h}$ electronic state of an atom can be given as

$$
\begin{aligned}
A_{J} & =\frac{1}{I J}\left\langle\Psi_{J}\left|H_{\mathrm{hfs}}\right| \Psi_{J}\right\rangle \\
& =\frac{\vec{\mu}_{k}}{I J} \cdot\left\langle\Psi_{J}\left|\sum_{i}^{n}\left(\frac{\vec{\alpha}_{i} \times \vec{r}_{i}}{r_{i}^{3}}\right)\right| \Psi_{J}\right\rangle,
\end{aligned}
$$

where, $I$ is the nuclear spin quantum number and $\Psi_{J}$ is the wavefunction of the $J^{t h}$ electronic state.

\section{COMPUTATIONAL DETAILS}

TABLE I: Basis and cutoff used for the atomic calculation.

\begin{tabular}{lcr}
\hline \hline Atom & Basis & Virtual cutoff (a.u.) \\
\hline $\mathrm{Li}$ & aug-cc-pCVQZ & \\
$\mathrm{Na}$ & aug-cc-pCVQZ & 500 \\
$\mathrm{~K}$ & dyall.cv4z & 500 \\
$\mathrm{Rb}$ & dyall.cv3z & 40 \\
$\mathrm{Cs}$ & dyall.cv4z & 50 \\
$\mathrm{Fr}$ & dyall.cv3z & \\
$\mathrm{Be}^{+}$ & aug-cc-pCVQZ & \\
$\mathrm{Mg}^{+}$ & aug-cc-pCVQZ & 500 \\
$\mathrm{Ca}^{+}$ & dyall.cv4z & 100 \\
$\mathrm{Sr}^{+}$ & dyall.cv3z & 40 \\
$\mathrm{Ba}^{+}$ & dyall.cv4z & 50 \\
$\mathrm{Ra}^{+}$ & dyall.cv3z & \\
\hline \hline
\end{tabular}

The DIRAC10 program package [39] is used to solve the DHF equation and to construct the one-electron and two-electron matrix elements. The magnetic HFS integrals are extracted from a locally modified version of DIRAC10. Gaussian charge distribution is considered for the finite size of the nucleus where the the nuclear parameters are taken from Ref. [40]. Restricted kinetic balance [41] condition is used to link small and large component basis function. No virtual pair approximation (NVPA) is used to solve DHF equation. This means that the negative energy solutions are removed by using projection operator and only positive energy solutions are included in the correlation calculations. However, how to go beyond the no-pair approximation by accounting for correlation contributions of negative energy states has been discussed in depth in Ref. [42-44]. In our calculation, we have used aug-cc-pCVQZ basis $[45,46]$ for $\mathrm{Li}, \mathrm{Na}$, Be and $\mathrm{Mg}$ atoms and dyall.cv3z basis [47] for Rb, Sr, Fr and $\mathrm{Ra}$ atoms and dyall.cv4z [47] basis for $\mathrm{K}, \mathrm{Ca}$, Cs and $\mathrm{Ba}$ atoms. All electrons are considered for the correlation calculation of all systems. The cutoff used for the virtual orbitals are compiled in Table I. 


\section{RESULTS AND DISCUSSION}

TABLE II: Magnetic hyperfine coupling constant (in MHz) of ground state of atoms.

\begin{tabular}{|c|c|c|c|c|c|c|}
\hline \multirow[t]{2}{*}{ Atom } & \multirow[t]{2}{*}{$\mathrm{SCF}$} & \multirow{2}{*}{$\begin{array}{c}\text { ECC } \\
{[31]}\end{array}$} & \multirow[t]{2}{*}{ Z-vector } & \multirow[t]{2}{*}{ Expt. } & \multicolumn{2}{|c|}{$\delta \%$} \\
\hline & & & & & ECC & Z-vector \\
\hline${ }^{6} \mathrm{Li}$ & 107.2 & 149.3 & 148.3 & $152.1[48]$ & 1.9 & 2.6 \\
\hline${ }^{7} \mathrm{Li}$ & 283.2 & 394.3 & 391.6 & $401.7[48]$ & 1.9 & 2.6 \\
\hline${ }^{23} \mathrm{Na}$ & 630.6 & 861.8 & 861.4 & $885.8[48]$ & 2.8 & 2.8 \\
\hline${ }^{39} \mathrm{~K}$ & 151.0 & 223.5 & 226.6 & $230.8[48]$ & 3.3 & 1.9 \\
\hline${ }^{40} \mathrm{~K}$ & -187.7 & -277.9 & -281.8 & $-285.7[49]$ & 2.8 & 1.4 \\
\hline${ }^{41} \mathrm{~K}$ & 82.9 & 122.7 & 124.4 & $127.0[48]$ & 3.5 & 2.1 \\
\hline${ }^{85} \mathrm{Rb}$ & 666.9 & 972.5 & 986.5 & $1011.9[50]$ & 4.1 & 2.6 \\
\hline${ }^{87} \mathrm{Rb}$ & 2260.1 & 3295.7 & 3343.3 & $3417.3[51]$ & 3.7 & 2.2 \\
\hline${ }^{133} \mathrm{Cs}$ & 1495.5 & 2179.1 & 2218.4 & $2298.1[52]$ & 5.5 & 3.6 \\
\hline${ }^{223} \mathrm{Fr}$ & 5518.0 & & 7537.4 & $7654(2)[53]$ & & 1.5 \\
\hline${ }^{9} \mathrm{Be}^{+}$ & -498.8 & -614.6 & -612.9 & $-625.0[54]$ & 1.7 & 2.0 \\
\hline${ }^{25} \mathrm{Mg}^{+}$ & -466.7 & -581.6 & -584.8 & $-596.2[55]$ & 2.5 & 1.9 \\
\hline${ }^{43} \mathrm{Ca}^{+}$ & -606.2 & -794.9 & -801.5 & $-806.4[56]$ & 1.4 & 0.6 \\
\hline${ }^{87} \mathrm{Sr}^{+}$ & -761.0 & -969.9 & -977.9 & $-1000.5(1.0)[57]$ & 3.2 & 2.3 \\
\hline${ }^{135} \mathrm{Ba}^{+}$ & 2737.4 & & 3513.3 & $3591.7[58]$ & & 2.2 \\
\hline${ }^{137} \mathrm{Ba}^{+}$ & 3062.1 & & 3930.2 & $4018.9[58]$ & & 2.3 \\
\hline${ }^{223} \mathrm{Ra}^{+}$ & 2842.8 & & 3446.3 & $3404(2)[59,60]$ & & 1.2 \\
\hline
\end{tabular}

In Table II, we present the magnetic HFS constant of alkali metal atoms and mono-positive alkaline earth metal ions in their ground state $\left({ }^{2} S\right)$ electronic configuration using the $\mathrm{Z}$-vector technique in the relativistic coupled-cluster framework. We have compiled the experimental values for these systems in the same table and the relative deviations of Z-vector results from the experimental values are presented as $\delta \%$. The results for different isotopes are calculated by using their corresponding nuclear magnetic moment values although the nuclear parameters in the nuclear model are taken as same for each isotope which is default the most stable isotopes in DIRAC10 [39]. Our calculated Z-vector results are in very good agreement with the experimental values. From the table, it is clear that the deviations of Z-vector results from the experiments are well within $3 \%$ except for the ${ }^{133} \mathrm{Cs}$ atom, where it is $3.6 \%$. The $\mathrm{Z}$-vector results are quite impressive, especially for the heavy atoms. The ECC values of magnetic HFS constant are taken from Ref. [31] and the deviations from the experiment are presented in the table. We have used same basis and cutoff for those systems for comparison purpose. The deviations of ECC and Z-vector values from the experimental values are presented in Fig. 1. From the figure, it is clear that Z-vector results are far better than the ECC results except for two small systems like $\mathrm{Li}$ and $\mathrm{Be}^{+}$. As the magnetic HFS constant is very sensitive to the near nuclear wavefunction, the above results show that the Zvector method can produce far better wavefunction in the nuclear region than the ECC method and the results are quite impressive for the heavy atoms. Although, ECC is a truncated series, in CCSD model, the natural truncation leads to very expensive terms. In Ref. [31], the truncation scheme proposed by Vaval et al are used to avoid the expensive terms in the ECC functional where

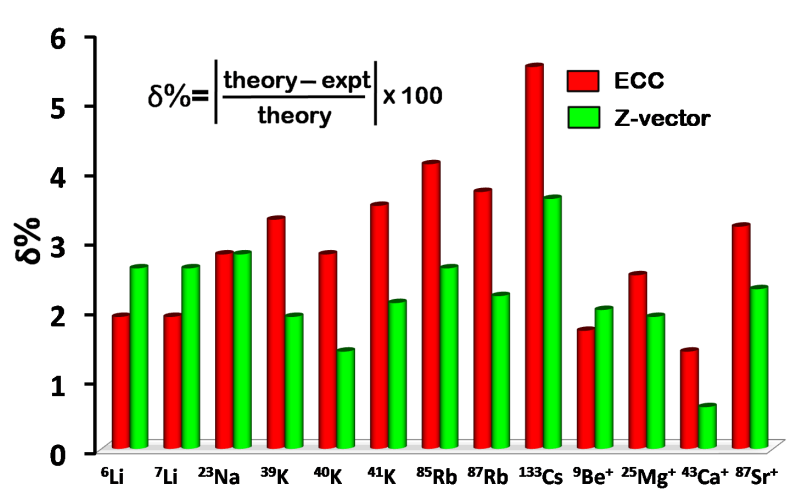

FIG. 1: Comparison of relative deviations between Z-vector and ECC values of magnetic HFS constant of atoms.

the right exponential is full within the CCSD approximation and the higher-order double-linked terms within the CCSD approximation are taken in the left exponent. This approximation introduces an additional error which may be the reason for the poor performance of ECC compared to Z-vector method.

TABLE III: Comparison of full CI and Z-vector magnetic HFS values (in $\mathrm{MHz}$ ) of ${ }^{7} \mathrm{Li}$

\begin{tabular}{lcr}
\hline \hline Basis & Full CI [31] & Z-vector \\
\hline aug-cc-pCVDZ & 384.1 & 383.9 \\
aug-cc-pCVTZ & 402.0 & 401.3 \\
aug-cc-pCVQZ & 386.0 & 385.2 \\
\hline \hline
\end{tabular}

${ }^{a}$ Considering 3 electrons and 189 virtual orbitals

TABLE IV: Comparison of full CI and Z-vector magnetic HFS values (in $\mathrm{MHz}$ ) of ${ }^{9} \mathrm{Be}^{+}$

\begin{tabular}{lcr}
\hline \hline Basis & Full CI [31] & Z-vector \\
\hline aug-cc-pCVDZ & -586.6 & -586.5 \\
aug-cc-pCVTZ & -615.7 & -615.4 \\
aug-cc-pCVQZ $^{a}$ & -613.0 & -612.7 \\
\hline \hline
\end{tabular}

${ }^{a}$ Considering 3 electrons and 183 virtual orbitals

The HFS constant predominantly depends on the spin density of the valence electron in the nuclear region and thus is not very sensitive to the retardation and magnetic effects described by the Breit interaction [61, 62]. It can be seen from the previous calculations that the higher order relativistic effects on these types of properties generally lie $\sim 0.5-1 \%$ [63-65]. It is worth to mention here that although we have correlated all electrons in our Zvector calculations, the results are not completely free from the uncertainty associated with core correlation as the $\mathrm{cvNz}(\mathrm{N}=3,4)$ basis set misses some important core correlating functions. The 1s-3d electrons also need much higher virtual energy orbitals for proper correlation functions as shown in Ref. [66, 67]. A series of calculations 
are done to estimate the uncertainty associated with the $\mathrm{Z}$-vector values of the magnetic HFS constant of these systems. The comparison between full configuration interaction (FCI) and Z-vector magnetic HFS constant values of ${ }^{7} \mathrm{Li}$ and ${ }^{9} \mathrm{Be}^{+}$has been made and is presented in Tables III and IV, respectively. By comparing Z-vector values with FCI values and considering all other sources of error like higher order relativistic effects, missing correlation effects etc, it can be assumed that the overall uncertainty in our final results is less than $5 \%$.

\section{CONCLUSION}

We have calculated the magnetic HFS constant of alkali metal atoms ( $\mathrm{Li}, \mathrm{Na}, \mathrm{K}, \mathrm{Rb}, \mathrm{Cs}$ and $\mathrm{Fr}$ ) and monopositive alkaline earth metal ions $\left(\mathrm{Be}^{+}, \mathrm{Mg}^{+}, \mathrm{Ca}^{+}, \mathrm{Sr}^{+}\right.$, $\mathrm{Ba}^{+}$and $\mathrm{Ra}^{+}$) using the Z-vector technique in the relativistic coupled-cluster framework. We have compared the Z-vector values and the ECC values taken from Ref. [31] with experiment and the comparison shows that the Z-vector method with single and double approximation can produce much accurate wavefunction in the nuclear region than the ECC method with the given approximation. A possible explanation for the poor performance of the ECC method is also given.

\section{Acknowledgement}

Author thanks Prof. Sourav Pal, Dr. Malaya K. Nayak, Dr. Nayana Vaval, Dr. Himadri Pathak for their valuable comments and insights in this work. Author acknowledges Dr. Malaya K. Nayak for providing the hyperfine integrals. Author acknowledges the resources of the Center of Excellence in Scientific Computing at CSIR-NCL. S.S. acknowledges the CSIR for the SPM fellowship.
[1] I. Lindgren and J. Morrison, Atomic Many-Body Theory (Springer-Verlag, New York, 1985).

[2] http://tf.nist.gov/cesium/atomichistory.htm.

[3] C. Champenois et al., Physics Letters A 331, 298 (2004).

[4] G. P. Barwood, G. Huang, H. A. Klein, P. Gill, and R. B. M. Clarke, Physical Review A 59, R3178 (1999).

[5] H. Margolis et al., Science 306, 1355 (2004).

[6] J. A. Sherman, W. Trimble, S. Metz, W. Nagourney, and N. Fortson, Progress on indium and barium single ion optical frequency standards, in LEOS Summer Topical Meetings, 2005 Digest of the, pp. 99-100, IEEE, 2005.

[7] R. Ozeri, W. M. Itano, R. B. Blakestad, J. Britton, J. Chiaverini, J. D. Jost, C. Langer, D. Leibfried, R. Reichle, S. Seidelin, J. H. Wesenberg, and D. J. Wineland, Physical Review A 75, 042329 (2007).

[8] C. F. Roos et al., Science 304, 1478 (2004).

[9] V. Flambaum and I. Khriplovich, Sov. Phys.-JETP (Engl. Transl.);(United States) 52 (1980).

[10] V. Flambaum, I. Khriplovich, and O. Sushkov, Physics Letters B 146, 367 (1984).

[11] T. Das, Hyperfine Interactions 34, 149 (1987).

[12] M. G. Kozlov, A. V. Titov, N. S. Mosyagin, and P. V. Souchko, Physical Review A 56, R3326 (1997).

[13] S. Porsev, Y. G. Rakhlina, and M. Kozlov, Journal of Physics B: Atomic, Molecular and Optical Physics 32, 1113 (1999).

[14] A. V. Titov, Y. V. Lomachuk, and L. V. Skripnikov, Phys. Rev. A 90, 052522 (2014).

[15] J. Čížek, Journal of Chemical Physics 45, 4256 (1966).

[16] J. Cizek, Advances in Chemical Physics: Correlation Effects in Atoms and Molecules (Wiley, Hoboken, NJ, 1967).

[17] R. J. Bartlett and G. D. Purvis, International Journal of Quantum Chemistry 14, 561 (1978).

[18] W. Kutzelnigg, Methods of Electronic Structure Theory (Plenum, New York, 1977).

[19] S. Pal, M. D. Prasad, and D. Mukherjee, Theoretica chimica acta 62, 523 (1983).

[20] S. Pal, Theoretica chimica acta 66, 207 (1984).
[21] K. Tanaka and H. Terashima, Chemical physics letters 106, 558 (1984).

[22] M. R. Hoffmann and J. Simons, Journal of Chemical Physics 88, 993 (1988).

[23] R. J. Bartlett, S. A. Kucharski, and J. Noga, Chemical physics letters 155, 133 (1989).

[24] S. Pal, M. D. Prasad, and D. Mukherjee, Pramana 18, 261 (1982).

[25] S. Pal, Theoretica chimica acta 66, 151 (1984).

[26] R. J. Bartlett and J. Noga, Chemical physics letters 150, 29 (1988).

[27] K. B. Ghose, P. G. Nair, and S. Pal, Chemical physics letters 211, 15 (1993).

[28] J. Arponen, Annals of Physics 151, 311 (1983).

[29] R. Bishop, J. Arponen, and P. Pajanne, Aspects of Manybody Effects in Molecules and Extended Systems, Lecture Notes in Chemistry Vol. 50 (Springer-Verlag, Berlin, 1989).

[30] P. G. Szalay, M. Nooijen, and R. J. Bartlett, Journal of Chemical Physics 103, 281 (1995).

[31] S. Sasmal, H. Pathak, M. K. Nayak, N. Vaval, and S. Pal, Phys. Rev. A 91, 022512 (2015).

[32] H. J. Monkhorst, Int. J. Quantum Chem. 12, 421 (1977).

[33] H. Sekino and R. J. Bartlett, International Journal of Quantum Chemistry 26, 255 (1984).

[34] N. C. Handy and H. F. Schaefer, Journal of Chemical Physics 81, 5031 (1984).

[35] E. A. Salter, G. W. Trucks, and R. J. Bartlett, Journal of Chemical Physics 90, 1752 (1989).

[36] S. Sasmal, H. Pathak, M. K. Nayak, N. Vaval, and S. Pal, Phys. Rev. A 91, 030503 (2015).

[37] A. Shee, L. Visscher, and T. Saue, The Journal of Chemical Physics 145, 184107 (2016).

[38] H. Koch et al., Journal of Chemical Physics 92, 4924 (1990).

[39] DIRAC, a relativistic ab initio electronic structure program, Release DIRAC10 (2010), written by T. Saue, L. Visscher and H. J. Aa. Jensen, with contributions from R. Bast, K. G. Dyall, U. Ekström, E. Eliav, T. Enevold- 
sen, T. Fleig, A. S. P. Gomes, J. Henriksson, M. Iliaš, Ch. R. Jacob, S. Knecht, H. S. Nataraj, P. Norman, J. Olsen, M. Pernpointner, K. Ruud, B. Schimmelpfennig, J. Sikkema, A. Thorvaldsen, J. Thyssen, S. Villaume, and S. Yamamoto (see http://www.diracprogram.org).

[40] L. Visscher and K. Dyall, Atomic Data and Nuclear Data Tables 67, 207 (1997).

[41] K. Faegri Jr and K. G. Dyall, Introduction to relativistic quantum chemistry (Oxford University Press, USA, 2007).

[42] W. Liu and I. Lindgren, Journal of Chemical Physics 139, 014108 (2013).

[43] W. Liu, Physics Reports 537, 59 (2014).

[44] W. Liu, National Science Review 3, 204 (2016).

[45] T. H. Dunning, Journal of Chemical Physics 90, 1007 (1989).

[46] D. E. Woon and T. H. Dunning Jr. (unpublished).

[47] K. G. Dyall, The Journal of Physical Chemistry A 113, 12638 (2009).

[48] A. Beckmann, K. Böklen, and D. Elke, Zeitschrift für Physik 270, 173 (1974).

[49] J. Eisinger, B. Bederson, and B. Feld, Physical Review 86, 73 (1952).

[50] J. Vanier, J.-F. Simard, and J.-S. Boulanger, Physical Review A 9, 1031 (1974).

[51] L. Essen, E. Hope, and D. Sutcliffe, Nature 189, 298 (1961).

[52] E. Arimondo, M. Inguscio, and P. Violino, Reviews of Modern Physics 49, 31 (1977).

[53] J. E. Sansonetti, Journal of physical and chemical reference data 36, 497 (2007).

[54] D. J. Wineland, J. J. Bollinger, and W. M. Itano, Phys- ical Review Letters 50, 628 (1983)

[55] W. M. Itano and D. J. Wineland, Physical Review A 24, 1364 (1981).

[56] F. Arbes, M. Benzing, T. Gudjons, F. Kurth, and G. Werth, Zeitschrift für Physik D Atoms, Molecules and Clusters 31, 27 (1994).

[57] F. Buchinger, E. B. Ramsay, E. Arnold, W. Neu, R. Neugart, K. Wendt, R. E. Silverans, P. Lievens, L. Vermeeren, D. Berdichevsky, R. Fleming, D. W. L. Sprung, and G. Ulm, Physical Review C 41, 2883 (1990).

[58] S. Trapp et al., Hyperfine Interactions 127, 57 (2000).

[59] K. Wendt et al., Zeitschrift für Physik D Atoms, Molecules and Clusters 4, 227 (1987).

[60] W. Neu et al., Zeitschrift für Physik D Atoms, Molecules and Clusters 11, 105 (1989).

[61] H. M. Quiney, J. K. Laerdahl, K. Fægri Jr, and T. Saue, Physical Review A 57, 920 (1998).

[62] E. Lindroth, B. Lynn, and P. Sandars, Journal of Physics B: Atomic, Molecular and Optical Physics 22, 559 (1989).

[63] S. A. Blundell, W. R. Johnson, and J. Sapirstein, Physical review letters 65, 1411 (1990).

[64] M. G. Kozlov, S. G. Porsev, and I. I. Tupitsyn, Physical review letters $\mathbf{8 6}, 3260$ (2001).

[65] V. A. Dzuba, V. V. Flambaum, and J. S. M. Ginges, Physical Review D 66, 076013 (2002).

[66] L. V. Skripnikov, The Journal of Chemical Physics 145, 214301 (2016).

[67] L. V. Skripnikov, D. E. Maison, and N. S. Mosyagin, Physical Review A 95, 022507 (2017). 